\title{
Generalized Entropy Power Inequalities and Monotonicity Properties of Information*
}

\author{
Mokshay Madiman and Andrew Barron \\ Department of Statistics \\ Yale University \\ 24 Hillhouse Avenue \\ New Haven, CT 06511, USA. \\ Email: mokshay.madiman, andrew.barron @yale.edu
}

\begin{abstract}
New families of Fisher information and entropy power inequalities for sums of independent random variables are presented. These inequalities relate the information in the sum of $n$ independent random variables to the information contained in sums over subsets of the random variables, for an arbitrary collection of subsets. As a consequence, a simple proof of the monotonicity of information in central limit theorems is obtained, both in the setting of i.i.d. summands as well as in the more general setting of independent summands with variance-standardized sums.
\end{abstract}

\section{Introduction}

Let $X_{1}, X_{2}, \ldots, X_{n}$ be independent random variables with densities and finite variances. Let $H$ denote the entropy, i.e., if $f$ is the probability density function of $X$, then $H(X)=$ $-E[\log f(X)]$. The classical entropy power inequality of Shannon [22] and Stam [25] states

$$
e^{2 H\left(X_{1}+\ldots+X_{n}\right)} \geq \sum_{i=1}^{n} e^{2 H\left(X_{j}\right)}
$$

Recently, Artstein, Ball, Barthe and Naor [1] proved a new entropy power inequality

$$
e^{2 H\left(X_{1}+\ldots+X_{n}\right)} \geq \frac{1}{n-1} \sum_{i=1}^{n} e^{2 H\left(\sum_{j \neq i} X_{j}\right)},
$$

where each term involves the entropy of the sum of $n-1$ of the variables excluding the $i$-th. It is not hard to see, by repeated application of (2) for a succession of values of $n$, that (2) in fact implies the inequality (4) and hence (11). We will present below a generalized entropy power inequality that subsumes both (2) and (10) and also implies several other interesting

*A summary of these results was submitted to the IEEE Symposium on Information Theory 2006 on January 9, 2006. The results were also announced on February 10, 2006 at the Inaugural Workshop for the Center for Information Theory and Applications in San Diego. 
inequalities. Remarkably, we provide simple and easily interpretable proofs of all of these inequalities, including of the monotonicity of entropy in central limit theorems.

More specifically, if $\mathcal{S}$ is an arbitrary collection of subsets of $\{1,2, \ldots, n\}$, we show that

$$
e^{2 H\left(X_{1}+\ldots+X_{n}\right)} \geq \frac{1}{r(\mathcal{S})} \sum_{\mathbf{S} \in \mathcal{S}} e^{2 H\left(\sum_{j \in \mathbf{S}} X_{j}\right)}
$$

where $r(\mathcal{S})$ is the maximum number of sets in $\mathcal{S}$ in which any one index appears. In particular, note that

1. Choosing $\mathcal{S}$ to be the class $\mathcal{S}_{1}$ of all singletons yields $r=1$ and hence (11).

2. Choosing $\mathcal{S}$ to be the class $\mathcal{S}_{n-1}$ of all sets of $n-1$ elements yields $r=n-1$ and hence (2).

3. Choosing $\mathcal{S}$ to be the class $\mathcal{S}_{m}$ of all sets of $m$ elements yields $r=\left(\begin{array}{c}n-1 \\ m-1\end{array}\right)$ and hence the inequality

$$
\exp \left\{2 H\left(X_{1}+\ldots+X_{n}\right)\right\} \geq \frac{1}{\left(\begin{array}{c}
n-1 \\
m-1
\end{array}\right)} \sum_{\mathbf{S} \in \mathcal{S}_{m}} \exp \left\{2 H\left(\sum_{i \in \mathbf{S}} X_{i}\right)\right\} .
$$

4. Choosing $\mathcal{S}$ to be the class of all sets of $k$ consecutive integers yields $r=k$ and hence the inequality

$$
\exp \left\{2 H\left(\frac{X_{1}+\ldots+X_{n}}{\sqrt{n}}\right)\right\} \geq \frac{1}{k} \sum_{\mathbf{S} \in \mathcal{S}} \exp \left\{2 H\left(\sum_{i \in \mathbf{S}} X_{i}\right)\right\} .
$$

In general, the inequality (3) clearly yields a whole family of useful entropy power inequalities, for arbitrary collections of subsets. Furthermore, equality holds in any of these inequalities if and only if the $X_{i}$ are normally distributed and the collection $\mathcal{S}$ is "nice" in a sense that will be made precise later.

These inequalities are relevant for the examination of monotonicity in central limit theorems. Indeed, if $X_{1}$ and $X_{2}$ are independent and identically distributed (i.i.d.), then (11) is equivalent to

$$
H\left(\frac{X_{1}+X_{2}}{\sqrt{2}}\right) \geq H\left(X_{1}\right)
$$

by using the scaling $H(a X)=H(X)+\log |a|$. This fact implies that the entropy of the standardized sums $Y_{n}=\frac{\sum_{i=1}^{n} X_{i}}{\sqrt{n}}$ increases along the powers-of-2 subsequence, i.e., $H\left(Y_{2^{k}}\right)$ is non-decreasing in $k$. Characterization of the increase in entropy in (6) was used in proofs of central limit theorems by Shimizu [23, Barron [3] and Johnson and Barron [13. In particular, Barron [3] showed that the sequence $\left\{H\left(Y_{n}\right)\right\}$ of entropies of the normalized sums converges to the entropy of the normal; this, incidentally, is equivalent to the convergence to 0 of the relative entropy (Kullback divergence) from a normal distribution when the $X_{i}$ have zero mean.

In 2004, Artstein, Ball, Barthe and Naor [1] (hereafter denoted by ABBN 1]) showed that $H\left(Y_{n}\right)$ is in fact a non-decreasing sequence for every $n$, solving a long-standing conjecture. In fact, (2) is equivalent in the i.i.d. case to the monotonicity property

$$
H\left(\frac{X_{1}+\ldots+X_{n}}{\sqrt{n}}\right) \geq H\left(\frac{X_{1}+\ldots+X_{n-1}}{\sqrt{n-1}}\right) .
$$


Note that the presence of the factor $n-1$ (rather than $n$ ) in the denominator of (2) is crucial for this monotonicity.

Likewise, for sums of independent random variables, our inequality (3) is equivalent to "monotonicity on average" properties for certain standardizations; for instance,

$$
H\left(\frac{X_{1}+\ldots+X_{n}}{\sqrt{n}}\right) \geq \frac{1}{\left(\begin{array}{c}
n \\
m
\end{array}\right)} \sum_{\mathbf{s} \in \mathcal{S}_{m}} H\left(\frac{\sum_{i \in \mathbf{S}} X_{i}}{\sqrt{m}}\right) .
$$

A similar monotonicity also holds, as we shall show, for arbitrary collections $\mathcal{S}$ and even when the sums are standardized by their variances. Here again the factor $r(\mathcal{S})$ (rather than the cardinality $|\mathcal{S}|$ of the collection) in the denominator of (3) for the unstandardized version is crucial.

\section{Outline of our development.}

We find that the key inequality (3) (and hence all of the above inequalities) as well as corresponding inequalities for Fisher information can be be proved by simple tools. Two of these tools, a convolution identity for score functions and the relationship between Fisher information and entropy (discussed in Section 2), are familiar in past work on entropy power inequalities. An additional trick is needed to obtain the denominator of $r(\mathcal{S})$ in (3). This is a simple variance drop inequality for statistics expressible via sums of functions of subsets of a collection of variables, particular cases of which are familiar in other statistical contexts (as we shall discuss). Such a variance drop was first used for information inequality development in $\mathrm{ABBN}$ [1. The variational characterization of Fisher information that is an essential ingredient of $\mathrm{ABBN}[1$ is not needed in our proofs.

For clarity of presentation, we find it convenient to first outline the proof of (17) for i.i.d. random variables. Thus, in Section [3] we establish the monotonicity result (17) in a simple and revealing manner that boils down to the geometry of projections (conditional expectations). Whereas $\mathrm{ABBN}$ [1] requires that $X_{1}$ has a $C^{2}$ density for monotonicity of Fisher divergence, absolute continuity of the density suffices in our approach. Furthermore, whereas the recent preprint of Shlyakhtenko 24] proves the analogue of the monotonicity fact for non-commutative or "free" probability theory, his method implies a proof for the classical case only assuming finiteness of all moments, while our direct proof requires only finite variance assumptions. Our proof also reveals in a simple manner the cases of equality in (7) (c.f., Schultz [21]). Although we do not write it out for brevity, the monotonicity of entropy for standardized sums of $d$-dimensional random vectors has an identical proof.

We recall that for a random variable $X$ with density $f$, the entropy is $H(X)=-E[\log f(X)]$. For a differentiable density, the score function is $\rho_{X}(x)=\frac{\partial}{\partial x} \log f(x)$, and the Fisher information is $I(X)=E\left[\rho_{X}^{2}(X)\right]$. They are linked by an integral form of the de Bruijn identity due to Barron [3], which permits certain convolution inequalities for $I$ to translate into corresponding inequalities for $H$.

Underlying our inequalities is the demonstration for independent, not necessarily identically distributed (i.n.i.d.) random variables with absolutely continuous densities that

$$
I\left(X_{1}+\ldots+X_{n}\right) \leq r(\mathcal{S}) \sum_{\mathbf{S} \in \mathcal{S}} w_{\mathbf{S}}^{2} I\left(\sum_{i \in \mathbf{S}} X_{i}\right)
$$

for any non-negative weights $w_{\mathbf{s}}$ that add to 1 over all subsets $\mathbf{s} \subset\{1, \ldots, n\}$ in $\mathcal{S}$. See Section 4 for details. Optimizing over $w$ yields an inequality for inverse Fisher information 
that extends the original inequality of Stam:

$$
\frac{1}{I\left(X_{1}+\ldots+X_{n}\right)} \geq \frac{1}{r(\mathcal{S})} \sum_{\mathbf{S} \in \mathcal{S}} \frac{1}{I\left(\sum_{i \in \mathbf{S}} X_{i}\right)} .
$$

Alternatively, using a scaling property of Fisher information to re-express our core inequality (9), we see that the Fisher information of the sum is bounded by a convex combination of Fisher informations of scaled partial sums:

$$
I\left(X_{1}+\ldots+X_{n}\right) \leq \sum_{\mathbf{S} \in \mathcal{S}} w_{\mathbf{S}} I\left(\frac{\sum_{i \in \mathbf{S}} X_{i}}{\sqrt{w_{\mathbf{S}} r(\mathcal{S})}}\right) .
$$

This integrates to give an inequality for entropy that is an extension of the "linear form of the entropy power inequality" developed by Dembo et al [7. Specifically we obtain

$$
H\left(X_{1}+\ldots+X_{n}\right) \leq \sum_{\mathbf{S} \in \mathcal{S}} w_{\mathbf{S}} H\left(\frac{\sum_{i \in \mathbf{S}} X_{i}}{\sqrt{w_{\mathbf{S}} r(\mathcal{S})}}\right) .
$$

See Section 5 for details. Likewise using the scaling property of entropy on (12) and optimizing over $w$ yields our extension of the entropy power inequality

$$
\exp \left\{2 H\left(X_{1}+\ldots+X_{n}\right)\right\} \geq \frac{1}{r(\mathcal{S})} \sum_{\mathbf{S} \in \mathcal{S}} \exp \left\{2 H\left(\sum_{j \in \mathbf{S}} X_{j}\right)\right\},
$$

described in Section [6.

Section 7 applies the preceding study to linear combinations of random variables, which is the relevant setting for studying central limit theorems for non-identically distributed random variables. In particular, we show that "entropy is monotone on average" in this setting.

Section 8 concludes with some discussion on the interpretation of our results and methods.

\section{Form of the inequalities.}

Both inverse Fisher information and entropy power satisfy an inequality of the form

$$
r(\mathcal{S}) \psi\left(X_{1}+\ldots+X_{n}\right) \geq \sum_{\mathbf{S} \in \mathcal{S}} \psi\left(\sum_{i \in \mathbf{S}} X_{i}\right) .
$$

We motivate the form (14) using the following almost trivial fact. Let $[n]=\{1,2, \ldots, n\}$.

Fact 1. For arbitrary numbers $\left\{a_{i}: i \in[n]\right\}$,

$$
\sum_{\mathbf{S} \in \mathcal{S}} \sum_{i \in \mathbf{S}} a_{i}=r(\mathcal{S}) \sum_{i \in[n]} a_{i}
$$

if each index $i$ appears in $\mathcal{S}$ the same number of times.

Indeed,

$$
\sum_{\mathbf{S} \in \mathcal{S}} \sum_{i \in \mathbf{S}} a_{i}=\sum_{i \in[n]} \sum_{\mathbf{S} \ni i, \mathbf{S} \in \mathcal{S}} a_{i}=\sum_{i \in[n]} r(\mathcal{S}) a_{i}=r(\mathcal{S}) \sum_{i \in[n]} a_{i} .
$$


If Fact 1 is thought of as $\mathcal{S}$-additivity of the sum function for real numbers, then (10) and (13) represent the $\mathcal{S}$-superadditivity of inverse Fisher information and entropy power functionals respectively with respect to convolution of the arguments. In the case of normal random variables, the inverse Fisher information and the entropy power equal the variance. Thus in that case (10) and (13) become Fact 1 with $a_{i}$ equal to the variance of $X_{i}$.

\section{Score Functions and Projections}

We use $\rho_{X}(x)=\frac{f^{\prime}(x)}{f(x)}$ to denote the (almost everywhere defined) score function of the random variable $X$ with absolutely continuous probability density function $f$. The score function $\rho_{X}(x)$ has zero mean, and its variance is just the Fisher information $I(X)$.

The first tool we need is a projection property of score functions of sums of independent random variables, which is well-known for smooth densities (c.f., Blachman [4]). For completeness, we give the proof. As shown by Johnson and Barron [13, it is sufficient that the densities are absolutely continuous; see 13] [Appendix 1] for an explanation of why this is so.

Lemma 1 (CONVOLUTION IDENTITY FOR SCORES). If $V_{1}$ and $V_{2}$ are independent random variables, and $V_{1}$ has an absolutely continuous density with score $\rho_{1}$, then $V_{1}+V_{2}$ has the score

$$
\rho(v)=E\left[\rho_{1}\left(V_{1}\right) \mid V_{1}+V_{2}=v\right]
$$

Proof. Let $f_{1}$ and $f_{V}$ be the densities of $V_{1}$ and $V=V_{1}+V_{2}$ respectively. Then, either bringing the derivative inside the integral for the smooth case, or via the more general formalism in [13,

$$
\begin{aligned}
f^{\prime}(v) & =\frac{\partial}{\partial v} E\left[f_{1}\left(v-V_{2}\right)\right] \\
& =E\left[f_{1}^{\prime}\left(v-V_{2}\right)\right] \\
& =E\left[f_{1}\left(v-V_{2}\right) \rho_{1}\left(v-V_{2}\right)\right]
\end{aligned}
$$

so that

$$
\begin{aligned}
\rho(v) & =\frac{f^{\prime}(v)}{f(v)} \\
& =E\left[\frac{f_{1}\left(v-V_{2}\right)}{f(v)} \rho_{1}\left(v-V_{2}\right)\right] \\
& =E\left[\rho_{1}\left(V_{1}\right) \mid V_{1}+V_{2}=v\right] .
\end{aligned}
$$

The second tool we need is a "variance drop lemma", the idea of which goes back at least to Hoeffding's seminal work [10] on $U$-statistics (see his Theorem 5.2). The following conventions are useful:

- $[n]$ is the index set $\{1,2, \ldots, n\}$. 
- For any $\mathbf{s} \subset[n], X_{\mathbf{S}}$ stands for the collection of random variables $\left(X_{i}: i \in \mathbf{s}\right)$, with the indices taken in their natural (increasing) order.

- We say that a function $f: \mathbb{R}^{d} \rightarrow \mathbb{R}$ is an additive function if there exist functions $f_{i}: \mathbb{R} \rightarrow \mathbb{R}$ such that $f\left(x_{1}, \ldots, x_{d}\right)=\sum_{i \in[d]} f_{i}\left(x_{i}\right)$.

- For $\psi^{(\mathbf{s})}: \mathbb{R}^{|\mathbf{S}|} \rightarrow \mathbb{R}$, we write $\psi^{(\mathbf{s})}\left(X_{\mathbf{S}}\right)$ for a function of $X_{\mathbf{S}}$ for any $\mathbf{s} \subset[n]$, so that $\psi^{(\mathbf{S})}\left(X_{\mathbf{S}}\right) \equiv \psi^{(\mathbf{S})}\left(X_{k_{1}}, \ldots, X_{k_{\mid \mathbf{S}} \mid}\right)$, where $k_{1}<k_{2}<\ldots<k_{|\mathbf{S}|}$ are the ordered indices in s.

The following two notions are not required for the inequalities we present, but help to clarify the cases of equality.

- A collection $\mathcal{S}$ of subsets of $[n]$ is said to be discriminating if for any distinct indices $i$ and $j$ in $[n]$, there is a set in $\mathcal{S}$ that contains $i$ but not $j$. Note that all the collections introduced in Section 1 were discriminating.

- A collection $\mathcal{S}$ of subsets of $[n]$ is said to be balanced if each index $i$ in $[n]$ appears in the same number (namely, $r(\mathcal{S})$ ) of sets in $\mathcal{S}$.

Lemma 2 (VARIANCE DROP). Suppose we are given a class of functions $\psi^{(\mathbf{S})}: \mathbb{R}^{|\mathbf{S}|} \rightarrow \mathbb{R}$ for any $\mathbf{s} \in \mathcal{S}$, where $\mathcal{S}$ is a given collection of subsets of $[n]$. Suppose also that $E \psi^{(\mathbf{S})}\left(X_{\mathbf{S}}\right)=0$ for each $\mathbf{s} \in \mathcal{S}$. Define

$$
U\left(X_{1}, \ldots, X_{n}\right)=\sum_{\mathbf{S} \in \mathcal{S}} w_{\mathbf{s}} \psi^{(\mathbf{s})}\left(X_{\mathbf{S}}\right)
$$

where $w=\left\{w_{\mathbf{s}}: \mathbf{s} \in \mathcal{S}\right\}$ is a probability distribution on $\mathcal{S}$. Then

$$
E U^{2} \leq r(\mathcal{S}) \sum_{\mathbf{S} \in \mathcal{S}} w_{\mathbf{S}}^{2} E\left[\psi^{(\mathbf{s})}\left(X_{\mathbf{S}}\right)\right]^{2} .
$$

When $\mathcal{S}$ is a discriminating collection, equality can hold only if each $\psi^{(\mathbf{S})}$ is an additive function.

Proof. Let $\bar{E}_{\mathbf{t}}$ be the ANOVA projection onto the set $\mathbf{t}$ (see the Appendix for details). Since

$$
\begin{aligned}
E U^{2} & =E\left[\sum_{\mathbf{s} \in \mathcal{S}} w_{\mathbf{s}} \sum_{\mathbf{t} \subset \mathbf{S}} \bar{E}_{\mathbf{t}} \psi^{(\mathbf{s})}\left(X_{\mathbf{S}}\right)\right]^{2} \\
& =E\left[\sum_{\mathbf{t}} \sum_{\mathbf{s} \supset \mathbf{t}, \mathbf{S} \in \mathcal{S}} w_{\mathbf{S}} \bar{E}_{\mathbf{t}} \psi^{(\mathbf{S})}\left(X_{\mathbf{S}}\right)\right]^{2}
\end{aligned}
$$

The outer summation over $\mathbf{t}$ can be restricted to non-empty $\mathbf{t}$, since $\bar{E}_{\phi}$ has no effect in the summation due to $\psi^{(\mathbf{S})}$ having zero mean. Thus, any given $\mathbf{t}$ in the expression has at least one element, and hence at least one element of $\mathbf{s} \supset \mathbf{t}$ is fixed. This means that the number of terms in the inner sum is upper bounded by $r(\mathcal{S})$, since that is the maximum number of sets 
in $\mathcal{S}$ that a fixed index can appear in. Thus using the orthogonality of the decomposition twice and the Cauchy-Schwartz inequality,

$$
\begin{aligned}
E U^{2} & =\sum_{\mathbf{t}} E\left[\sum_{\mathbf{s} \supset \mathbf{t}, \mathbf{s} \in \mathcal{S}} w_{\mathbf{S}} \bar{E}_{\mathbf{t}} \psi^{(\mathbf{s})}\left(X_{\mathbf{S}}\right)\right]^{2} \\
& \leq \sum_{\mathbf{t}} r(\mathcal{S}) \sum_{\mathbf{s} \supset \mathbf{t}, \mathbf{s} \in \mathcal{S}} w_{\mathbf{S}}^{2} E\left[\bar{E}_{\mathbf{t}} \psi^{(\mathbf{s})}\left(X_{\mathbf{S}}\right)\right]^{2} \\
& =r(\mathcal{S}) \sum_{\mathbf{S} \in \mathcal{S}} w_{\mathbf{S}}^{2} E\left[\psi^{(\mathbf{s})}\left(X_{\mathbf{S}}\right)\right]^{2}
\end{aligned}
$$

which proves the inequality.

Now suppose $\psi^{\left(\mathbf{S}^{\prime}\right)}$ is not additive. This means that for some set $\mathbf{t} \subset \mathbf{s}^{\prime}$ with at least two elements, $\bar{E}_{\mathbf{t}} \psi^{\left(\mathbf{S}^{\prime}\right)}\left(X_{\mathbf{S}^{\prime}}\right) \neq 0$. For this choice of $\mathbf{t}$, the inner sum in the inequality (23) runs over strictly fewer than $r(\mathcal{S})$ subsets $\mathbf{s}$ if $\mathcal{S}$ is a discriminating collection. In particular, the inequality (23) must be strict. Thus each $\psi^{(\mathbf{S})}$ must be an additive function if equality holds, i.e., it must be composed only of main effects and no interactions.

Remark 1 . Suppose $\psi: \mathbb{R}^{m} \rightarrow \mathbb{R}$ is symmetric in its arguments, and $E \psi\left(X_{1}, \ldots, X_{m}\right)=0$. Define

$$
U\left(X_{1}, \ldots, X_{n}\right)=\frac{1}{\left(\begin{array}{c}
n \\
m
\end{array}\right)} \sum_{\{\mathbf{s} \subset[n]:|\mathbf{S}|=m\}} \psi\left(X_{\mathbf{S}}\right),
$$

Then Hoeffding [10] showed

$$
E U^{2} \leq \frac{m}{n} E \psi^{2}
$$

which is implied by Lemma 2 under the symmetry assumptions. In statistical language, $U$ defined in (24) is a $U$-statistic of degree $m$ with symmetric, mean zero kernel $\psi$ that is applied to data of sample size $n$. Thus (25) quantitatively captures the reduction of variance of a $U$-statistic when sample size $n$ increases. For $m=1$, this is the trivial fact that the empirical variance of a function based on i.i.d. samples is the actual variance scaled by $n^{-1}$. For $m>1$, the functions $\psi\left(X_{\mathbf{S}}\right)$ are no longer independent, nevertheless the variance of the $U$-statistic drops by a factor of $\frac{m}{n}$.

Remark 2. Not only is our proof valid for the more general non-symmetric case, it also seems to illuminate the underlying statistical idea (the ANOVA decomposition) as well as the underlying geometry (Hilbert space projections) better than Hoeffding's original combinatorial proof. In 8], Efron and Stein assert in their Comment 3 that an ANOVA-like decomposition "yields one-line proofs of Hoeffding's important theorems 5.1 and 5.2"; presumably our proof of Lemma 2 is a generalization of what they had in mind.

The third key tool in our approach to monotonicity is the well-known link between Fisher information and entropy, whose origin is the de Bruijn identity first described by Stam [25]. The standard way of obtaining entropy inequalities from Fisher information inequalities relies on adding a normal with the same variance, and is now a standard method to "lift" results 
from Fisher information to entropy, after the integral form of the de Bruijn identity was proved by Barron 3. For our purpose, we need a modified inequality that does not restrict the variance.

Lemma 3. Let $X$ be a random variable with a density and finite variance. Suppose $X_{t} \stackrel{(d)}{=} X+$ $\sqrt{t} Z$, where $Z$ is a standard normal independent of $X$. Then,

$$
H(X)=\frac{1}{2} \log (2 \pi e)-\frac{1}{2} \int_{0}^{\infty}\left[I\left(X_{t}\right)-\frac{1}{1+t}\right] d t
$$

Proof. The identity is valid in the case that the variances of $Z^{\prime}$ and $X$ match. Indeed, Barron [2] shows that

$$
H(X)=\frac{1}{2} \log (2 \pi e v)-\frac{1}{2} \int_{0}^{\infty}\left[I\left(X_{t}\right)-\frac{1}{v+t}\right] d t .
$$

(We applied a change of variables using $t=\tau v$ to Equation (2.23) in [2]). This has the advantage of positivity of the integrand but the disadvantage that is seems to depend on $v$. One can use

$$
\log v=\int_{0}^{\infty}\left[\frac{1}{1+t}-\frac{1}{v+t}\right]
$$

to re-express it in the form (26).

Remark 3 . Thus any convex inequality for $I$ immediately implies the corresponding inequality for $H$ even if the variances are not the same, where by convex inequality is meant one in which an information is greater than the a sum of informations with weights that add to 1 .

\section{Monotonicity in the IID case}

For i.i.d. random variables, inequalities (2) and (4) reduce to the monotonicity $H\left(Y_{n}\right) \geq$ $H\left(Y_{m}\right)$ for $n>m$, where

$$
Y_{n}=\frac{1}{\sqrt{n}} \sum_{i=1}^{n} X_{i} .
$$

For clarity of presentation of ideas, we focus first on the i.i.d. case, beginning with Fisher information.

Proposition 1 (Monotonicity of Fisher Information). If $X_{i}$ are i.i.d. random variables, and $Y_{n-1}$ has an absolutely continuous density, then

$$
I\left(Y_{n}\right) \leq I\left(Y_{n-1}\right)
$$

with equality iff $X_{1}$ is normal or $I\left(Y_{n}\right)=\infty$. 
Proof. We use the following notational conventions: The (unnormalized) sum is $S_{n}=\sum_{i \in[n]} X_{i}$, and the leave-one-out sum leaving out $X_{j}$ is $S^{(j)}=\sum_{i \neq j} X_{i}$. Setting $\rho_{j}=\rho_{S^{(j)}}\left(S^{(j)}\right)$, we have

$$
\begin{aligned}
\rho_{S_{n}}\left(S_{n}\right) & \stackrel{(a)}{=} E\left[\rho_{j} \mid S_{n}\right] \\
& \stackrel{(b)}{=} \frac{1}{n} \sum_{j=1}^{n} E\left[\rho_{j} \mid S_{n}\right] \\
& =\frac{1}{n} E\left[\sum_{j=1}^{n} \rho_{j} \mid S_{n}\right] .
\end{aligned}
$$

Here, (a) follows from application of Lemma 1 to $S_{n}=S^{(j)}+X_{j}$, while (b) follows from symmetry. Since the length of a vector is not less than the length of its projection (i.e., by Cauchy-Schwartz inequality),

$$
I\left(S_{n}\right)=E\left[\rho_{S_{n}}\left(S_{n}\right)\right]^{2} \leq \frac{1}{n^{2}} E\left[\sum_{j=1}^{n} \rho_{j}\right]^{2} .
$$

Lemma 2 yields

$$
E\left[\sum_{j \in[n]} \rho_{j}\right]^{2} \leq(n-1) \sum_{j \in[n]} E\left[\rho_{j}\right]^{2}=(n-1) n I\left(S_{n-1}\right),
$$

so that

$$
I\left(S_{n}\right) \leq \frac{n-1}{n} I\left(S_{n-1}\right) .
$$

If $X^{\prime}=a X$, then $\rho_{X^{\prime}}\left(X^{\prime}\right)=\frac{1}{a} \rho_{X}(X)$ and $a^{2} I\left(X^{\prime}\right)=I(X)$; hence

$$
I\left(Y_{n}\right)=n I\left(S_{n}\right) \leq(n-1) I\left(S_{n-1}\right)=I\left(Y_{n-1}\right) .
$$

The inequality implied by Lemma 2 can be tight only if each $\rho_{j}$ is an additive function, but we already know that $\rho_{j}$ is a function of the sum. The only functions that are both additive and functions of the sum are linear functions of the sum; hence the two sides of (30) can be finite and equal only if the score $\rho_{j}$ is linear, i.e., if all the $X_{i}$ are normal. It is trivial to check that $X_{1}$ normal or $I\left(Y_{n}\right)=\infty$ imply equality.

The monotonicity result for entropy in the i.i.d. case now follows by combining Proposition 1 and Lemma 3

Theorem 1 ((Monotonicity of Entropy: IID CASE)). Suppose $X_{i}$ are i.i.d. random variables with densities. Suppose $X_{1}$ has finite variance, and

$$
Y_{n}=\frac{1}{\sqrt{n}} \sum_{i=1}^{n} X_{i}
$$

Then

$$
H\left(Y_{n}\right) \geq H\left(Y_{n-1}\right) .
$$

The two sides are finite and equal iff $X_{1}$ is normal. 
Remark 4. As discussed in the introduction, Proposition 1 and Theorem 1 were first obtained by ABBN [1] by somewhat more elaborate means. Here we see that they can be obtained by familar projection properties of Fisher information and the variance drop lemma.

After the submission of these results to ISIT 2006, we became aware of a contemporary and independent development of the simple proof of the monotonicity fact (Theorem 1) by Tulino and Verdú [27. In their work they take nice advantage of projection properties through minimum mean squared error interpretations. It is pertinent to note that the proofs of Theorem 1 (in 27] and in this paper) share essentials, because of the following observations.

Consider estimation of a random variable $X$ from an observation $Y=X+Z$ in which an independent standard normal $Z$ has been added. Then the score function of $Y$ is related to the difference between two predictors of $\mathrm{X}$ (maximum likelihood and Bayes), i.e.,

$$
-\rho(Y)=Y-E[X \mid Y]
$$

and hence the Fisher information $I(Y)=E \rho^{2}(Y)$ is the same as the mean square difference $E\left[(Y-E[X \mid Y])^{2}\right]$, or equivalently, by the Pythagorean identity,

$$
I(Y)=\operatorname{Var}(Z)-E\left[(X-E[X \mid Y])^{2}\right] .
$$

Thus the Fisher information (entropy derivative) is related to the minimal mean squared error. These (and more general) identities relating differences between predictors to scores and relating their mean squared errors to Fisher informations are developed in statistical decision theory in the work of Stein and Brown. These developments are described, for instance, in the point estimation text by Lehmann and Casella [16] [Chapters 4.3 and 5.5], in their study of Bayes risk, admissibility, and, in some cases minimaxity, of conditional means $E[X \mid Y]$.

Tulino and Verdú [27] emphasize the minimal mean squared error property of the entropy derivative and associated projection properies that (along with the variance drop inequality which they note in the leave one out case) also give Proposition 1 and Theorem 1 That is a nice idea. Working directly with the minimal mean squared error as the entropy derivative they bypass the use of Fisher information. In the same manner Verdú and Guo [28] give an alternative proof of the entropy power inequality. If one takes note of the above identities one sees that their proofs and ours are substantially the same, except that the same quantities are given alternative interpretations in the two works, and that we give extensions to arbitrary collections of subsets.

\section{Fisher Information Inequalities}

In this section, we demonstrate our core inequality (9).

Proposition 2. Let $\left\{X_{i}\right\}$ be independent random variables with densities and finite variances. Define

$$
T_{n}=\sum_{i \in[n]} X_{i} \quad \text { and } \quad T^{(\mathbf{S})}=\sum_{i \in \mathbf{S}} X_{i}
$$

for each $\mathbf{s} \in \mathcal{S}$, where $\mathcal{S}$ is an arbitrary collection of subsets of $[n]$. Let $w$ be any probability distribution on $\mathcal{S}$. If each $T^{(\mathbf{S})}$ has an absolute continuous density, then

$$
I\left(T_{n}\right) \leq r(\mathcal{S}) \sum_{\mathbf{s} \in \mathcal{S}} w_{\mathbf{s}}^{2} I\left(T^{(\mathbf{s})}\right)
$$


where $w_{\mathbf{s}}=w(\{\mathbf{s}\})$. When $\mathcal{S}$ is discriminating, both sides can be finite and equal only if each $X_{i}$ is normal.

Proof. In the sequel, for convenience, we abuse notation by using $\rho$ to denote several different score functions; $\rho(Y)$ always means $\rho_{Y}(Y)$. For each $j$, Lemma 1 and the fact that $T^{(\mathbf{S})}$ has an absolutely continuous density imply

$$
\rho\left(T_{n}\right)=E\left[\rho\left(\sum_{i \in \mathbf{S}} X_{i}\right) \mid T_{n}\right] .
$$

Taking a convex combinations of these identities gives, for any $\left\{w_{\mathbf{s}}\right\}$ such that $\sum_{\mathbf{s} \in \mathcal{S}} w_{\mathbf{S}}=1$,

$$
\begin{aligned}
\rho\left(T_{n}\right) & =\sum_{\mathbf{S} \in \mathcal{S}} w_{\mathbf{S}} E\left[\rho\left(\sum_{i \in \mathbf{S}} X_{i}\right) \mid T_{n}\right] \\
& =E\left[\sum_{\mathbf{S} \in \mathcal{S}} w_{\mathbf{s}} \rho\left(T^{(\mathbf{S})}\right) \mid T_{n}\right] .
\end{aligned}
$$

By applying the Cauchy-Schwartz inequality and Lemma 2 in succession, we get

$$
\begin{aligned}
I\left(T_{n}\right) & \leq E\left[\sum_{\mathbf{S} \in \mathcal{S}} w_{\mathbf{s}} \rho\left(T^{(\mathbf{S})}\right)\right]^{2} \\
& \leq r(\mathcal{S}) \sum_{\mathbf{S} \in \mathcal{S}} E\left[w_{\mathbf{S}} \rho\left(T^{(\mathbf{s})}\right)\right]^{2} \\
& =r(\mathcal{S}) \sum_{\mathbf{s} \in \mathcal{S}} w_{\mathbf{S}}^{2} I\left(T^{(\mathbf{s})}\right) .
\end{aligned}
$$

The application of Lemma 2 can yield equality only if each $\rho\left(T^{(\mathbf{S})}\right)$ is additive; since the score $\rho\left(T^{(\mathbf{S})}\right)$ is already a function of the sum $T^{(\mathbf{S})}$, it must in fact be a linear function, so that each $X_{i}$ must be normal.

Naturally, it is of interest to minimize the upper bound of Proposition 2 over the weighting distribution $w$, which is easily done either by an application of Jensen's inequality for the reciprocal function, or by the method of Lagrange multipliers. Optimization of the bound implies that Proposition 2 is equivalent to the following Fisher information inequalities.

Theorem 2. Let $\left\{X_{i}\right\}$ be independent random variables such that each $T_{m}^{(\mathbf{S})}$ has an absolutely continuous density. Then

$$
\frac{1}{I\left(T_{n}\right)} \geq \frac{1}{r(\mathcal{S})} \sum_{\mathbf{S} \in \mathcal{S}} \frac{1}{I\left(T_{m}^{(\mathbf{S})}\right)} .
$$

When $\mathcal{S}$ is discriminating, the two sides are positive and equal iff each $X_{i}$ is normal and $\mathcal{S}$ is also balanced. 
Theorem 2 for the special case $\mathcal{S}=\mathcal{S}_{1}$ of singleton sets is sometimes known as the "Stam inequality" and has a long history. Stam [25] was the first to prove Proposition 2 for $\mathcal{S}_{1}$, and he credited his doctoral advisor de Bruijn with noticing the equivalence to Theorem 2 for $\mathcal{S}_{1}$. Subsequently several different proofs have appeared: in Blachman 4] using Lemma 10 in Carlen [5] using "superadditivity" of the Fisher information, and in Kagan [14] as a consequence of an inequality for Pitman estimators. On the other hand, the special case of the leave-one-out sets $\mathcal{S}=\mathcal{S}_{n-1}$ in Theorem 2 was first pointed out in ABBN [1]. Zamir [30] used data processing properties of the Fisher information to prove some different extensions of the $\mathcal{S}_{1}$ case, including a multivariate version; see also Liu and Viswanath 18, for some related interpretations. Our result for arbitrary collections of subsets is new; yet our proof of this general result is essentially no harder than the elementary proofs of the original inequality by Stam and Blachman.

\section{$5 \quad$ Entropy Inequalities}

Proposition 2 can be viewed as a generalized form of the traditional convolution inequality

$$
I\left(V_{1}+V_{2}\right) \leq \alpha^{2} I\left(V_{1}\right)+(1-\alpha)^{2} I\left(V_{2}\right),
$$

which is implied by Lemma In that case, scaling the random variables led to

$$
I\left(\sqrt{\alpha} V_{1}+\sqrt{1-\alpha} V_{2}\right) \leq \alpha I\left(V_{1}\right)+(1-\alpha) I\left(V_{2}\right)
$$

which has a convex combination of Fisher informations on the right side. In our more general setting, we develop an inequality (48), which is analogous to this inequality in certain respects.

Proposition 3 (EnTropy of Sums). Let $X_{i}$ be independent random variables with densities. Then, for any probability distribution $w$ on $\mathcal{S}$,

$$
\begin{aligned}
H\left(\sum_{i \in[n]} X_{i}\right) \geq \sum_{\mathbf{S} \in \mathcal{S}} & w_{\mathbf{S}} H\left(\sum_{i \in \mathbf{S}} X_{i}\right) \\
& +\frac{1}{2} H(w)-\frac{1}{2} \log r(\mathcal{S}) .
\end{aligned}
$$

When $\mathcal{S}$ is discriminating, equality can hold only if each $X_{i}$ is normal.

Proof. As pointed out earlier, Proposition 2 is equivalent to

$$
I\left(T_{n}\right) \leq \sum_{\mathbf{S} \in \mathcal{S}} w_{\mathbf{S}} I\left(\frac{\sum_{i \in \mathbf{S}} X_{i}}{\sqrt{w_{\mathbf{S}} r(\mathcal{S})}}\right)
$$

Using Lemma 3 leads to

$$
\begin{aligned}
H\left(T_{n}\right) & \geq \sum_{\mathbf{S} \in \mathcal{S}} w_{\mathbf{S}} H\left(\frac{\sum_{i \in \mathbf{S}} X_{i}}{\sqrt{w_{\mathbf{S}} r(\mathcal{S})}}\right) \\
& =\sum_{\mathbf{S} \in \mathcal{S}} w_{\mathbf{S}} H\left(\sum_{i \in \mathbf{S}} X_{i}\right)-\frac{1}{2} \sum_{\mathbf{S} \in \mathcal{S}} w_{\mathbf{S}} \log w_{\mathbf{S}}-\frac{1}{2} \log r(\mathcal{S}),
\end{aligned}
$$

which proves the desired result. 
Let $Y_{n}=\frac{T_{n}}{\sqrt{n}}$ and $Y_{m}^{(\mathbf{S})}=\frac{T_{m}^{(\mathbf{S})}}{\sqrt{m}}$ be the scaled sums. Then Proposition 3, applied to the collection $\mathcal{S}_{m}=\{\mathbf{s} \subset[n]:|\mathbf{s}|=m\}$, implies

$$
H\left(Y_{n}\right) \geq \sum_{\mathbf{S} \in \mathcal{S}_{m}} w_{\mathbf{s}} H\left(Y_{m}^{(\mathbf{s})}\right)-\frac{1}{2}\left[\log \left(\begin{array}{c}
n \\
m
\end{array}\right)-H(w)\right] .
$$

The term on the right indicates that we pay a cost for deviations of the weighting distribution $w$ from the uniform. In particular, choosing $w$ to be uniform implies that entropy is "monotone on average" with uniform weights for scaled sums of i.n.i.d. random variables.

Corollary 1. Suppose $X_{i}$ are independent random variables with densities. Then

$$
H\left(Y_{n}\right) \geq \frac{1}{\left(\begin{array}{c}
n \\
m
\end{array}\right)} \sum_{\mathbf{s} \in \mathcal{S}_{m}} H\left(Y_{m}^{(\mathbf{s})}\right) .
$$

Remark 5. It is interesting to contrast Corollary 1]with the results of Han 9] (see also Cover and Thomas [6] [Section 16.5]), where it is shown that the per-variable joint entropy decreases monotonically in the size of the subset. In particular, with no assumptions on $\left(X_{1}, \ldots, X_{n}\right)$ except that they have a joint density,

$$
\frac{H\left(X_{[n]}\right)}{n} \leq \frac{1}{\left(\begin{array}{c}
n \\
m
\end{array}\right)} \sum_{\mathbf{S} \in \mathcal{S}_{m}} \frac{H\left(X_{\mathbf{S}}\right)}{m},
$$

where $H\left(X_{\mathbf{S}}\right)$ is the joint entropy of $X_{\mathbf{S}}$. For independent random variables, this holds with equality, and simply follows from Fact 1 .

\section{Entropy power inequalities}

Proposition 3 is equivalent to a hierarchy of entropy power inequalities for independent collections. Recall that the entropy power

$$
N(X)=\frac{e^{2 H(X)}}{2 \pi e}
$$

is the variance of the normal with the same entropy as $X$. Note that we have loosely used the term "entropy power inequality" even when the constant factor of $2 \pi e$ is not included.

Theorem 3. For independent random variables with finite variances,

$$
N\left(\sum_{i \in[n]} X_{i}\right) \geq \frac{1}{r(\mathcal{S})} \sum_{\mathbf{S} \in \mathcal{S}} N\left(\sum_{i \in \mathbf{S}} X_{i}\right) .
$$

When $\mathcal{S}$ is discriminating, the two sides are equal iff each $X_{i}$ is normal and $\mathcal{S}$ is also balanced. 
Proof. Define

$$
Z=\sum_{\mathbf{s} \in \mathcal{S}} N\left(T^{(\mathbf{s})}\right)
$$

and

$$
\mu(\mathbf{s})=\frac{N\left(T^{(\mathbf{s})}\right)}{Z}
$$

Writing $N^{(\mathbf{s})}=N\left(T^{(\mathbf{S})}\right)$ and $w(\mathbf{s})=w_{\mathbf{s}}$, Proposition 3 implies that

$$
\begin{aligned}
H\left(T_{n}\right) & \geq \sum_{\mathbf{S} \in \mathcal{S}} w(\mathbf{s}) \cdot \frac{1}{2} \log N^{(\mathbf{s})}+\frac{1}{2}[H(w)-\log r(\mathcal{S})] \\
& =\frac{1}{2} \sum_{\mathbf{S} \in \mathcal{S}} w(\mathbf{s}) \log [\mu(\mathbf{s}) Z]+\frac{1}{2}[H(w)-\log r(\mathcal{S})],
\end{aligned}
$$

so that

$$
\begin{aligned}
2 H\left(T_{n}\right) \geq & =\log \frac{Z}{r(\mathcal{S})}-[H(w)+D(w \| \mu)]+[H(w)] \\
& =\log \frac{Z}{r(\mathcal{S})}-D(w \| \mu) .
\end{aligned}
$$

Exponentiating gives

$$
N\left(T_{n}\right) \geq r(\mathcal{S})^{-1} e^{-D(w \| \mu)} Z .
$$

It remains to optimize the right side over $w$, which is done by setting $w=\mu$ since $D(w \| \mu) \geq 0$. The necessary conditions for equality follow from that for Proposition [3 and it is easily checked using Fact 1 that this is also sufficient. The proof is complete.

Remark 6. The traditional Shannon inequality involving the entropy powers of the summands 22] as well as the inequality of ABBN [1] involving the entropy powers of the "leave-one-out" normalized sums are two special cases of Theorem [3. corresponding to $\mathcal{S}=\mathcal{S}_{1}$ and $\mathcal{S}=\mathcal{S}_{n-1}$. Proofs of the former subsequent to Shannon's include those of Stam [25], Blachman [4, Lieb [17] (using the Hausdorff-Young inequality), and Dembo, Cover and Thomas [7. Note that unlike the previous proofs of these special cases, our proof of the equivalence between the linear form of Proposition [3] and Theorem 3 reduces to the non-negativity of the relative entropy.

As in Section [5. we also present a corollary for scaled sums, which trivially follows from Theorem 3 .

Corollary 2. Suppose $X_{i}$ are independent random variables with densities. Then, writing $Y_{n}=\frac{T_{n}}{\sqrt{n}}$ and $Y_{m}^{(\mathbf{S})}=\frac{T_{m}^{(\mathbf{S})}}{\sqrt{m}}$,

$$
N\left(Y_{n}\right) \geq \frac{1}{\left(\begin{array}{c}
n \\
m
\end{array}\right)} \sum_{\mathbf{s} \in \mathcal{S}_{m}} N\left(Y_{m}^{(\mathbf{s})}\right)
$$


Remark 7. Inspired by results of Han [9], Cover and Thomas [6] [Theorem 16.5.2] show that for any collection of random variables with a joint density,

$$
\left[N\left(X_{[n]}\right)\right]^{\frac{1}{n}} \leq \frac{1}{\left(\begin{array}{c}
n \\
m
\end{array}\right)} \sum_{\mathbf{S} \in \mathcal{S}_{m}}\left[N\left(X_{\mathbf{S}}\right)\right]^{\frac{1}{m}} .
$$

This is interesting to contrast with Corollary 2

\section{Entropy is Monotone on Average}

In this section, we consider linear combinations of independent random variables. This is motivated by the following consideration. Consider a series of independent random variables $\left\{X_{i}: i \in \mathbb{N}\right\}$ with zero mean and finite variances, $\sigma_{i}^{2}=\operatorname{Var}\left(X_{i}\right)$. The variance of the sum of $n$ variables is denoted $v_{n}=\sum_{i \in[n]} \sigma_{i}^{2}$, and the standardized sum is

$$
Y_{n}=\frac{\sum_{i \in[n]} X_{i}}{\sqrt{v_{n}}} .
$$

Lindeberg's condition states that

$$
\Lambda_{n}(\delta) \equiv \sum_{j} E\left[\left(\frac{X_{i}}{\sqrt{v_{n}}}\right)^{2} 1_{\left\{\left|\frac{X_{i}}{\sqrt{v_{n}}}\right|>\delta\right\}}\right] \rightarrow 0
$$

for any $\delta>0$. The Lindeberg-Feller CLT states that Lindeberg's condition is equivalent to $Y_{n} \Rightarrow N(0,1)$ and the individual smallness condition $\max _{i \in[n]} \frac{\sigma_{i}^{2}}{v_{n}} \rightarrow 0$. Is there an analogue of the monotonicity of information in this setting?

To address this question, we prove the following result, which applies to appropriately standardized linear combinations of independent random variables. Note that the symbol $T$ has a new definition below.

Proposition 4 (Monotonicity on AVERAGe). Suppose $\left\{X_{i}\right\}$ are independent random variables with densities and finite variances. Set

$$
T_{n}=\sum_{i=1}^{n} a_{i} X_{i}
$$

where $a$ is on the unit sphere, i.e., $\sum_{i=1}^{n} a_{i}^{2}=1$. For any $\mathbf{s} \in \mathcal{S}$, set $\bar{a}_{\mathbf{S}}=\left[\sum_{i \in \mathbf{S}} a_{i}^{2}\right]^{\frac{1}{2}}$, and define

$$
T^{(\mathbf{S})}=\frac{1}{\bar{a}_{\mathbf{S}}} \sum_{i \in \mathbf{S}} a_{i} X_{i}
$$

and

$$
\lambda(\mathbf{s})=\frac{\bar{a}_{\mathbf{S}}^{2}}{r(\mathcal{S})}
$$

Then, letting $\psi$ represent either the inverse Fisher information $I^{-1}$ or the entropy $H$ or the entropy power $N$,

$$
\psi\left(T_{n}\right) \geq \sum_{\mathbf{s} \in \mathcal{S}} \lambda(\mathbf{s}) \psi\left(T^{(\mathbf{s})}\right) .
$$

In each case, the two sides are finite and equal iff each $X_{i}$ is normal. 
Proof. Each result follows from the correponding result for sums by appropriate scalings. We illustrate this for entropy power and leave the statements for the other information quantities to the reader. Apply Theorem 3 to $X_{i}^{\prime}=a_{i} X_{i}$ to get

$$
\begin{aligned}
N\left(\sum_{i \in[n]} a_{i} X_{i}\right) & \geq \frac{1}{r(\mathcal{S})} \sum_{\mathbf{S} \in \mathcal{S}} N\left(\sum_{i \in \mathbf{S}} a_{i} X_{i}\right) \\
& =\frac{1}{r(\mathcal{S})} \sum_{\mathbf{S} \in \mathcal{S}} N\left(\bar{a}_{\mathbf{S}} T^{(\mathbf{S})}\right) \\
& =\sum_{\mathbf{S} \in \mathcal{S}} \lambda(\mathbf{s}) N\left(T^{(\mathbf{S})}\right),
\end{aligned}
$$

since $N(a X)=a^{2} N(X)$.

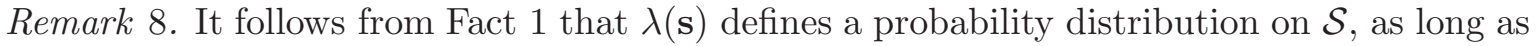
the collection $\mathcal{S}$ is balanced. This justifies the interpretation of Proposition 4 as displaying "monotonicity on average". The averaging distribution $\lambda$ is tuned to the linear combination of interest.

Theorem 4 (Monotonicity of Entropy: General Case). Let $\left\{X_{i}: i \in \mathbb{N}\right\}$ be independent random variables with densities. Suppose $X_{i}$ has zero mean and finite variance $\sigma_{i}^{2}$. Set $v_{n}=\sum_{i \in[n]} \sigma_{i}^{2}$ and $v^{(\mathbf{S})}=\sum_{i \in \mathbf{S}} \sigma_{i}^{2}$ for sets $\mathbf{s}$ in the collection $\mathcal{S}$. Define the standardized sums

$$
Y_{n}=\frac{\sum_{i \in[n]} X_{i}}{\sqrt{v_{n}}}
$$

and

$$
Y^{(\mathbf{s})}=\frac{\sum_{i \in \mathbf{S}} X_{i}}{\sqrt{v^{(\mathbf{S})}}}
$$

Then

$$
H\left(Y_{n}\right) \geq \sum_{\mathbf{s} \in \mathcal{S}} \lambda(\mathbf{s}) H\left(Y^{(\mathbf{s})}\right)
$$

where $\lambda(\mathbf{s})=\frac{1}{r(\mathcal{S})} \frac{v^{(\mathbf{S})}}{v_{n}}$. Furthermore, if $\mathcal{S}$ is discriminating, then the inequality is strict unless each $X_{i}$ is normal.

Proof. Set $X_{i}=\sigma_{i} X_{i}^{\prime}$, where each $X_{i}^{\prime}$ now has mean 0 and variance 1 . Choose $a_{i}=\frac{\sigma_{i}}{\sqrt{v_{n}}}$ so that $\sum_{i \in[n]} a_{i}^{2}=1$; then $\bar{a}_{\mathbf{S}}^{2}=\sum_{i \in \mathbf{S}} a_{i}^{2}=\frac{v^{(\mathbf{S})}}{v_{n}}$. Thus

$$
Y_{n}=\sum_{i \in[n]} \frac{X_{i}}{\sqrt{v_{n}}}=\sum_{i \in[n]} a_{i} X_{i}^{\prime},
$$

and

$$
Y_{m}^{(\mathbf{s})}=\sum_{i \in \mathbf{S}} \frac{X_{i}}{\sqrt{v^{(\mathbf{S})}}}=\frac{1}{\bar{a}_{\mathbf{S}}} \sum_{i \in \mathbf{S}} a_{i} X_{i}^{\prime} .
$$

Now an application of Proposition 4 gives the desired result. 
Remark 9. Theorem 4 did not require the Lindeberg condition, but the entropic LindebergFeller theorem $H\left(Y_{n}\right) \rightarrow \frac{1}{2} \log (2 \pi e)$ (proved by Johnson [12] under appropriate conditions) does. It would be of interest to obtain a direct proof of the the entropic Lindeberg-Feller theorem using the monotonicity combined with the Lindeberg condition.

\section{Conclusion}

We have laid out a number of information monotonicity facts above. A remarkable feature of our proofs is that their main ingredients (such as Lemmas 1 and 2) are rather well-known. Both our results as well as the proofs lend themselves to intuitive statistical interpretations, which we have pointed out in the paper.

Our results have interesting implications for the interpretation of central limit theorems. The Central Limit Theorem (CLT) in its simplest classical form states: Given i.i.d. random variables $X_{1}, X_{2}, \ldots, X_{n}, \ldots$ with mean 0 and variance 1 , the distributions of the standardized sums $Y_{n}=\frac{1}{\sqrt{n}} \sum_{i=1}^{n} X_{i}$ converge to the standard normal distribution $N(0,1)$. Information theory endows the CLT with strong intuitive and substantive content, as was noticed very early on. Indeed, the following two facts have been long known: (i) the normal distribution has the maximum entropy among all probability distributions with bounded variance, and (ii)

if $X_{1}$ and $X_{2}$ are i.i.d. with densities, then $H\left(\frac{X_{1}+X_{2}}{\sqrt{2}}\right) \geq H\left(X_{1}\right)$. The second fact implies that the entropy $H\left(Y_{n}\right)$ of the normalized sums is non-decreasing along the powers-of-2 subsequence. ABBN [1] showed (as do we) that $H\left(Y_{n}\right)$ is in fact a non-decreasing sequence for every $n$. The intuition is that the CLT captures a universal phenomenon for probability distributions with finite variance: namely, forming normalized sums yields random variables with increasing entropy, and this sequence of entropies converges to the maximum entropy possible, which is the entropy of the normal with that variance. In this sense, the CLT is a formulation of the "second law of thermodynamics" in physics. Even in the setting of variance-standardized sums (i.e., central limit theorems for independent but not identically distributed summands), a general monotonicity on average property holds with respect to normalized sums of an arbitrary collection of normalized subset sums. Thus, the "second law" interpretation of central limit theorems extends to the general case of independent summands.

In addition to nice interpretation of central limit theorems, the new Fisher information and entropy power inequalities we present are of intrinsic interest. Fisher information inequalities have interpretations in terms of the information that can be gained about an underlying location parameter from sums of random variables. Entropy power inequalities are useful not just to obtain bounds on entropy of sums, but have also been used to prove results about capacity of communication channels (starting with Shannon [22, also see Liu and Viswanath [19] and references therein for some recent developments from this point of view) and the logarithmic Sobolev inequality for the Gaussian distribution (implicitly by Stam [25], see also Carlen [5]). We remind the reader that our very general $\mathcal{S}$-superadditivity inequalities for Fisher information and entropy power hold for arbitrary collections $\mathcal{S}$, and specialize to both the Shannon-Stam inequalities and the inequalities of ABBN 1]. In particular, we prove all these inequalities transparently using only simple projection facts, a variance drop lemma and classical information-theoretic ideas. 


\section{A The Analysis of Variance Decomposition}

In order to prove the variance drop lemma, we use a decomposition of functions in $L^{2}\left(\mathbb{R}^{n}\right)$, which is nothing but the Analysis of Variance (ANOVA) decomposition of a statistic. For any $j \in[n], E_{j} \psi$ denotes the conditional expectation of $\psi$, given all random variables other than $X_{j}$, i.e.,

$$
E_{j} \psi\left(x_{1}, \ldots, x_{n}\right)=E\left[\psi\left(X_{1}, \ldots, X_{n}\right) \mid X_{i}=x_{i} \quad \forall i \neq j\right]
$$

averages out the dependence on the $j$-th coordinate.

Fact 2 (ANOVA Decomposition). Suppose $\psi: \mathbb{R}^{n} \rightarrow \mathbb{R}$ satisfies $E \psi^{2}\left(X_{1}, \ldots, X_{n}\right)<\infty$, i.e., $\psi \in L^{2}$, for independent random variables $X_{1}, X_{2}, \ldots, X_{n}$. For $\mathbf{s} \subset[n]$, define the orthogonal linear subspaces

$$
\mathcal{H}_{\mathbf{S}}=\left\{\psi \in L^{2}: E_{j} \psi=\psi 1_{\{j \notin \mathbf{S}\}} \forall j \in[n]\right\}
$$

of functions depending only on the variables indexed by $\mathbf{s}$. Then $L^{2}$ is the orthogonal direct sum of this family of subspaces, i.e., any $\psi \in L^{2}$ can be written in the form

$$
\psi=\sum_{\mathbf{S} \subset[n]} \psi_{\mathbf{s}}
$$

where $\psi_{\mathbf{s}} \in \mathcal{H}_{\mathbf{s}}$.

Let $E_{\mathbf{S}}$ denote the integrating out of the variables in $\mathbf{s}$, so that $E_{j}=E_{\{j\}}$. Keeping in mind that the order of integrating out independent variables does not matter (i.e., the $E_{j}$ are commuting projection operators in $L^{2}$ ), we can write

$$
\begin{aligned}
\phi & =\prod_{j=1}^{n}\left[E_{j}+\left(I-E_{j}\right)\right] \phi \\
& =\sum_{\mathbf{S} \subset[n]} \prod_{j \notin \mathbf{S}} E_{j} \prod_{j \in \mathbf{S}}\left(I-E_{j}\right) \phi \\
& =\sum_{\mathbf{S} \subset[n]} \phi_{\mathbf{S}},
\end{aligned}
$$

where

$$
\phi_{\mathbf{S}}=\bar{E}_{\mathbf{S}} \phi \equiv E_{\mathbf{S}^{c}} \prod_{j \notin \mathbf{S}}\left(I-E_{j}\right) \phi .
$$

In order to show that the subspaces $\mathcal{H}_{\mathbf{s}}$ are orthogonal, observe that for any $\mathbf{s}_{1}$ and $\mathbf{s}_{2}$, there is at least one $j$ such that $\mathbf{s}_{1} \subset \operatorname{Im}\left(E_{j}\right)$ and $\mathbf{s}_{2} \subset \operatorname{Im}\left(I-E_{j}\right)$; hence every vector in $\mathbf{s}_{1}$ is orthogonal to every vector in $\mathbf{s}_{2}$.

Remark 10. In the language of ANOVA familiar to statisticians, when $\phi$ is the empty set, $\psi_{\phi}$ is the mean; $\psi_{\{1\}}, \psi_{\{2\}}, \ldots, \psi_{\{n\}}$ are the main effects; $\left\{\psi_{\mathbf{s}}:|\mathbf{s}|=2\right\}$ are the pairwise interactions, and so on. Fact 2 implies that for any subset $\mathbf{s} \subset[n]$, the function $\sum_{\{R: R \subset \mathbf{s}\}} \psi_{R}$ is the best approximation (in mean square) to $\psi$ that depends only on the collection $X_{\mathbf{S}}$ of random variables. 
Remark 11. The historical roots of this decomposition lie in the work of von Mises [29] and Hoeffding [10. For various refinements and interpretations, see Kurkjian and Zelen [15], Jacobsen [11, Rubin and Vitale [20], and Efron and Stein [8]; these works include applications of such decompositions to experimental design, linear models, $U$-statistics, and jackknife theory. Takemura [26] describes a general unifying framework for ANOVA decompositions.

\section{References}

[1] S. Artstein, K. M. Ball, F. Barthe, and A. Naor. Solution of Shannon's problem on the monotonicity of entropy. J. Amer. Math. Soc., 17(4):975-982 (electronic), 2004.

[2] A.R. Barron. Monotonic central limit theorem for densities. Technical Report \#50, Department of Statistics, Stanford University, California, 1984.

[3] A.R. Barron. Entropy and the central limit theorem. Ann. Probab., 14:336-342, 1986.

[4] N.M. Blachman. The convolution inequality for entropy powers. IEEE Trans. Information Theory, IT-11:267-271, 1965.

[5] E. A. Carlen. Superadditivity of Fisher's information and logarithmic Sobolev inequalities. J. Funct. Anal., 101(1):194-211, 1991.

[6] T.M. Cover and J.A. Thomas. Elements of Information Theory. J. Wiley, New York, 1991.

[7] A. Dembo, T.M. Cover, and J.A. Thomas. Information-theoretic inequalities. IEEE Trans. Inform. Theory, 37(6):1501-1518, 1991.

[8] B. Efron and C. Stein. The jackknife estimate of variance. Ann. Stat., 9(3):586-596, 1981.

[9] Te Sun Han. Nonnegative entropy measures of multivariate symmetric correlations. Information and Control, 36(2):133-156, 1978.

[10] W. Hoeffding. A class of statistics with asymptotically normal distribution. Ann. Math. Stat., 19(3):293-325, 1948.

[11] R. L. Jacobsen. Linear Algebra and ANOVA. PhD thesis, Cornell University, 1968.

[12] O. Johnson. Entropy inequalities and the central limit theorem. Stochastic Process. Appl., 88:291-304, 2000.

[13] O. Johnson and A.R. Barron. Fisher information inequalities and the central limit theorem. Probab. Theory Related Fields, 129(3):391-409, 2004.

[14] A. Kagan. An inequality for the Pitman estimators related to the Stam inequality. Sankhyā Ser. A, 64:281-292, 2002.

[15] B. Kurkjian and M. Zelen. A calculus for factorial arrangements. Ann. Math. Statist., 33:600-619, 1962.

[16] E. L. Lehmann and G. Casella. Theory of Point Estimation. Springer Texts in Statistics. Springer-Verlag, New York, second edition, 1998. 
[17] E. H. Lieb. Proof of an entropy conjecture of Wehrl. Comm. Math. Phys., 62(1):35-41, 1978.

[18] T. Liu and P. Viswanath. Two proofs of the fisher information inequality via data processing arguments. Preprint, 2005.

[19] T. Liu and P. Viswanath. An extremal inequality motivated by multiterminal information theoretic problems. Preprint, 2006.

[20] H. Rubin and R. A. Vitale. Asymptotic distribution of symmetric statistics. Ann. Statist., 8(1):165-170, 1980.

[21] H. Schultz. Semicircularity, gaussianity and monotonicity of entropy. Preprint, 2005.

[22] C.E. Shannon. A mathematical theory of communication. Bell System Tech. J., 27:379423, 623-656, 1948.

[23] R. Shimizu. On Fisher's amount of information for location family. In G.P.Patil et al, editor, Statistical Distributions in Scientific Work, volume 3, pages 305-312. Reidel, 1975 .

[24] D. Shlyakhtenko. A free analogue of Shannon's problem on monotonicity of entropy. Preprint, 2005.

[25] A.J. Stam. Some inequalities satisfied by the quantities of information of Fisher and Shannon. Information and Control, 2:101-112, 1959.

[26] A. Takemura. Tensor analysis of ANOVA decomposition. J. Amer. Statist. Assoc., 78(384):894-900, 1983.

[27] A. M. Tulino and S. Verdú. Monotonic decrease of the non-gaussianness of the sum of independent random variables: A simple proof. Preprint, 2006.

[28] S. Verdú and D. Guo. A simple proof of the entropy-power inequality. Preprint, 2006.

[29] R. von Mises. On the asymptotic distribution of differentiable statistical functions. Ann. Math. Stat., 18(3):309-348, 1947.

[30] R. Zamir. A proof of the Fisher information inequality via a data processing argument. IEEE Trans. Inform. Theory, 44(3):1246-1250, 1998. 